\title{
BlackboxNLP 2021
}

Proceedings of the Fourth BlackboxNLP Workshop on Analyzing and Interpreting Neural Networks for NLP

November 11, 2021 
C2021 The Association for Computational Linguistics

Order copies of this and other ACL proceedings from:

Association for Computational Linguistics (ACL)

209 N. Eighth Street

Stroudsburg, PA 18360

USA

Tel: +1-570-476-8006

Fax: +1-570-476-0860

acleaclweb.org

ISBN 978-1-955917-06-3 


\section{Introduction}

BlackboxNLP is the workshop on analyzing and interpreting neural networks for NLP.

In the last few years, neural networks have rapidly become a central component in NLP systems. The improvement in accuracy and performance brought by the introduction of neural networks has typically come at the cost of our understanding of the system: How do we assess what the representations and computations are that the network learns? The goal of this workshop is to bring together people who are attempting to peek inside the neural network black box, taking inspiration from machine learning, psychology, linguistics, and neuroscience.

In this fourth edition of the workshop, hosted by the 2021 conference on Empirical Methods in Natural Language Processing (EMNLP), we accepted 41 archival papers and eight extended abstracts. The workshop also provided a platform for authors of four Findings of ACL papers, and seven Findings of EMNLP papers, to present their work as a poster at the workshop. In addition, for the first time, the workshop worked in collaboration with the ACL Rolling Review system (ARR), accepting two more archival papers through that avenue.

BlackboxNLP would not have been possible without the dedication of its program committee. We would like to thank them for their invaluable effort in providing timely and high-quality reviews on a short notice. We are also grateful to our invited speakers for contributing to our program.

Jasmijn Bastings, Yonatan Belinkov, Emmanuel Dupoux, Mario Giulianelli, Dieuwke Hupkes, Yuval Pinter and Hassan Sajjad 



\section{Organizers:}

Jasmijn Bastings, Google Amsterdam

Yonatan Belinkov, Technion - Israel Institute of Technology

Emmanuel Dupoux, Ecole Normale Supérieure and INRIA

Mario Giulianelli, University of Amsterdam

Dieuwke Hupkes, Facebook AI Research

Yuval Pinter, Ben-Gurion University of the Negev

Hassan Sajjad, Qatar Computing Research Institute

\section{Program Committee:}

Badr Abdullah - Saarland University

Heike Adel - Bosch Center for Artificial Intelligence

Sachin Agarwal - Apple

Željko Agić - Unity Technologies

Leila Arras - Fraunhofer Heinrich Hertz Institute

Kalyani Asthana - University of California Irvine

Pepa Atanasova - University of Copenhagen

Tania Avgustinova - Saarland University

Parsa Bagherzadeh - Concordia University

Niranjan Balasubramanian - Stony Brook University

Vinayshekhar Bannihatti Kumar - Amazon Web Services

Laurent Besacier - LIG

Nikolay Bogoychev - University of Edinburgh

Gemma Boleda - Universitat Pompeu Fabra / ICREA

Jonathan Brophy - University of Oregon

Lisa Bylinina - Bookarang

Stergios Chatzikyriakidis - University of Gothenburg

Hanjie Chen - University of Virginia

Ting-Rui Chiang - Carnegie Mellon University

Marta R. Costa-jussà - Universitat Politècnica de Catalunya

Benoit Crabbé - University of Paris

Verna Dankers - University of Edinburgh

Debajyoti Datta - University of Virginia

Roberto Dessì - Facebook AI Research Paris / UPF

Anne Dirkson - Leiden University

Radina Dobreva - University of Edinburgh

Yanai Elazar - Bar-Ilan University

Allyson Ettinger - University of Chicago

Jean-Philippe - Fauconnier Apple Inc.

Ghazi Felhi - Université Paris Nord

Antske Fokkens - VU Amsterdam

Robert Frank - Yale University

Richard Futrell - University of California, Irvine

Sebastian Gehrmann - Google Research

Michael Goodale - Ecole Normale Superieure, Department of Cognitive Studies

Loïc Grobol - École Normale Supérieure 
Prachi Gupta - New York University Tandon School of Engineering

David Harwath - The University of Texas at Austin

Peter Hase - University of North Carolina at Chapel Hill

Simon Hegelich - TUM

John Hewitt - Stanford University

Dewei $\mathrm{Hu}$ - University of Copenhagen

Cassandra L. Jacobs - University of Wisconsin, Madison

Alon Jacovi - Bar Ilan University

Robin Jia - Facebook AI Research

Zhiying Jiang - University of Waterloo

Richard Johansson - University of Gothenburg

Jaap Jumelet - University of Amsterdam

Lis Kanashiro Pereira - Ochanomizu University

Eugene Kharitonov - Facebook AI

Germán Kruszewski - Naver Labs Europe

Kunal Kukreja - University of Washington

Jenny Kunz - Linköping University

Shalom Lappin - King's College London

Alessandro Lenci - University of Pisa

Minghan Li - University of Waterloo

Sheng Liang - Center for Information and Language Processing, LMU Munich

Jindřich Libovický - Ludwig Maximilian University of Munich

Tomasz Limisiewicz - Charles University in Prague

Nelson F. Liu - Stanford University

David Mareček - Charles University

R. Thomas McCoy - Johns Hopkins University

Houman Mehrafarin - Iran University of Science and Technology

Koji Mineshima - Keio University

Saliha Muradoglu - The Australian National University

Anmol Nayak - ARiSE Labs at Bosch

Joakim Nivre - Uppsala University

Bhargavi Paranjape - University of Washington

Siddharth Patwardhan - Apple

Paolo Pedinotti - University of Pisa

Frithjof Petrick - RWTH Aachen University

Karl Pichotta - Memorial Sloan Kettering Cancer Center

Aman Priyanshu - Manipal Institute of Technology

Sara Rajaee - Iran University of Science and Technology

Abhilasha Ravichander - Carnegie Mellon University

Rudolf Rosa - Charles University, Faculty of Mathematics and Physics, Institute of Formal and

Applied Linguistics

Jan Rosendahl - RWTH Aachen University

Swarnadeep Saha - University of North Carolina at Chapel Hill

Wojciech Samek - Fraunhofer Heinrich Hertz Institute

Hendrik Schuff - Bosch Center for Artificial Intelligence

Robert Schwarzenberg - German Research Center For Artificial Intelligence (DFKI)

Djamé Seddah - Inria

Rico Sennrich - University of Zurich

Mattia Setzu - University of Pisa

Tatiana Shavrina - Sberbank, Sberdevices, HSE University

Sanchit Sinha - University of Virginia 
Pia Sommerauer - Vrije Universiteit Amsterdam

Vinitra Swamy - EPFL

Aarne Talman - University of Helsinki

Arka Talukdar - New York University

shimin tao - huawei

Jörg Tiedemann - University of Helsinki

Ivan Titov - University of Edinburgh / University of Amsterdam

Jithendra Vepa - Observe AI

Sarah Wiegreffe - Georgia Institute of Technology

Adina Williams - Facebook, Inc.

Hao Yang - Huawei Co. Ltd

Naoki Yoshinaga - Institute of Industrial Science, the University of Tokyo

Fabio Massimo Zanzotto - University of Rome Tor Vergata

Sheng Zhang - North Carolina State University

Yichu Zhou - University of Utah

\section{Invited Speakers:}

Sara Hooker, Google Brain

Ana Marasović, Allen AI

Willem Zuidema, University of Amsterdam 



\section{Table of Contents}

To what extent do human explanations of model behavior align with actual model behavior?

Grusha Prasad, Yixin Nie, Mohit Bansal, Robin Jia, Douwe Kiela and Adina Williams .........1

Test Harder than You Train: Probing with Extrapolation Splits

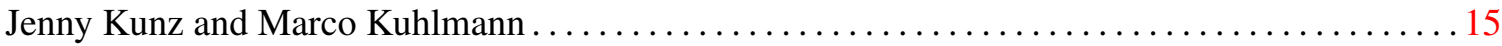

Does External Knowledge Help Explainable Natural Language Inference? Automatic Evaluation vs. Human Ratings

Hendrik Schuff, Hsiu-Yu Yang, Heike Adel and Ngoc Thang Vu....................... 26

The Language Model Understood the Prompt was Ambiguous: Probing Syntactic Uncertainty Through Generation

Laura Aina and Tal Linzen...

On the Limits of Minimal Pairs in Contrastive Evaluation

Jannis Vamvas and Rico Sennrich ..................................... 58

What Models Know About Their Attackers: Deriving Attacker Information From Latent Representations

Zhouhang Xie, Jonathan Brophy, Adam Noack, Wencong You, Kalyani Asthana, Carter Perkins, Sabrina Reis, Zayd Hammoudeh, Daniel Lowd and Sameer Singh . . . . . . . . . . . . . . . . . . . . 69

ALL Dolphins Are Intelligent and SOME Are Friendly: Probing BERT for Nouns' Semantic Properties and their Prototypicality

Marianna Apidianaki and Aina Garí Soler .

ProSPer: Probing Human and Neural Network Language Model Understanding of Spatial Perspective

Tessa Masis and Carolyn Anderson ........................................ 95

Can Transformers Jump Around Right in Natural Language? Assessing Performance Transfer from SCAN

Rahma Chaabouni, Roberto Dessì and Eugene Kharitonov

Transferring Knowledge from Vision to Language: How to Achieve it and how to Measure it?

Tobias Norlund, Lovisa Hagström and Richard Johansson . .

Discrete representations in neural models of spoken language

Bertrand Higy, Lieke Gelderloos, Afra Alishahi and Grzegorz Chrupała ................. 163

Word Equations: Inherently Interpretable Sparse Word Embeddings through Sparse Coding

Adly Templeton ................................................. 177

A howling success or a working sea? Testing what BERT knows about metaphors

Paolo Pedinotti, Eliana Di Palma, Ludovica Cerini and Alessandro Lenci................. 192

How Length Prediction Influence the Performance of Non-Autoregressive Translation?

Minghan Wang, GUO Jiaxin, Yuxia Wang, Yimeng Chen, Su Chang, Hengchao Shang, Min Zhang,

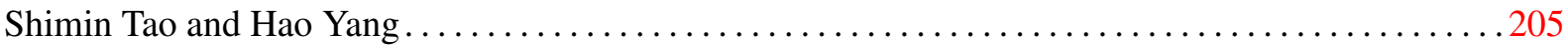

On the Language-specificity of Multilingual BERT and the Impact of Fine-tuning

Marc Tanti, Lonneke van der Plas, Claudia Borg and Albert Gatt ..................... 214 
Relating Neural Text Degeneration to Exposure Bias

Ting-Rui Chiang and Yun-Nung Chen $\ldots \ldots \ldots \ldots \ldots \ldots \ldots \ldots \ldots \ldots \ldots \ldots \ldots \ldots \ldots \ldots \ldots \ldots \ldots \ldots, 228$

Efficient Explanations from Empirical Explainers

Robert Schwarzenberg, Nils Feldhus and Sebastian Möller.

Variation and generality in encoding of syntactic anomaly information in sentence embeddings

Qinxuan $\mathrm{Wu}$ and Allyson Ettinger... 250

Enhancing Interpretable Clauses Semantically using Pretrained Word Representation

Rohan Kumar Yadav, Lei Jiao, Ole-Christoffer Granmo and Morten Goodwin ...

Analyzing BERT's Knowledge of Hypernymy via Prompting

Michael Hanna and David Mareček ...

An in-depth look at Euclidean disk embeddings for structure preserving parsing

Federico Fancellu, Lan Xiao, Allan Jepson and Afsaneh Fazly ... 283

Training Dynamic based data filtering may not work for NLP datasets

Arka Talukdar, Monika Dagar, Prachi Gupta and Varun Menon

Multi-Layer Random Perturbation Training for improving Model Generalization Efficiently

Lis Kanashiro Pereira, Yuki Taya and Ichiro Kobayashi. . .

Screening Gender Transfer in Neural Machine Translation

Guillaume Wisniewski, Lichao Zhu, Nicolas Bailler and François Yvon ...

What BERT Based Language Model Learns in Spoken Transcripts: An Empirical Study

Ayush Kumar, Mukuntha Narayanan Sundararaman and Jithendra Vepa ...

Assessing the Generalization Capacity of Pre-trained Language Models through Japanese Adversarial Natural Language Inference

Hitomi Yanaka and Koji Mineshima . . 337

Investigating Negation in Pre-trained Vision-and-language Models

Radina Dobreva and Frank Keller

Not all parameters are born equal: Attention is mostly what you need

Nikolay Bogoychev.

Not All Models Localize Linguistic Knowledge in the Same Place: A Layer-wise Probing on BERToids' Representations

Mohsen Fayyaz, Ehsan Aghazadeh, Ali Modarressi, Hosein Mohebbi and Mohammad Taher Pile-

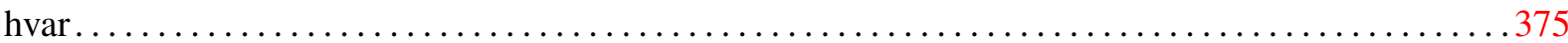

Learning Mathematical Properties of Integers

Maria Ryskina and Kevin Knight. .

Probing Language Models for Understanding of Temporal Expressions

Shivin Thukral, Kunal Kukreja and Christian Kavouras . .

How Familiar Does That Sound? Cross-Lingual Representational Similarity Analysis of Acoustic Word Embeddings

Badr Abdullah, Iuliia Zaitova, Tania Avgustinova, Bernd Möbius and Dietrich Klakow ... 407 
Perturbing Inputs for Fragile Interpretations in Deep Natural Language Processing

Sanchit Sinha, Hanjie Chen, Arshdeep Sekhon, Yangfeng Ji and Yanjun Qi ...

An Investigation of Language Model Interpretability via Sentence Editing

Samuel Stevens and $\mathrm{Yu} \mathrm{Su}$.

Interacting Knowledge Sources, Inspection and Analysis: Case-studies on Biomedical text processing

Parsa Bagherzadeh and Sabine Bergler.................................... 447

Attacks against Ranking Algorithms with Text Embeddings: A Case Study on Recruitment Algorithms

Anahita Samadi, debapriya banerjee and Shirin Nilizadeh. . .

Controlled tasks for model analysis: Retrieving discrete information from sequences

Ionut-Teodor Sorodoc, Gemma Boleda and Marco Baroni ...

The Acceptability Delta Criterion: Testing Knowledge of Language using the Gradience of Sentence Acceptability

Héctor Vázquez Martínez

How Does BERT Rerank Passages? An Attribution Analysis with Information Bottlenecks

Zhiying Jiang, Raphael Tang, Ji Xin and Jimmy Lin...

Do Language Models Know the Way to Rome?

Bastien Liétard, Mostafa Abdou and Anders Søgaard.

Exploratory Model Analysis Using Data-Driven Neuron Representations

Daisuke Oba, Naoki Yoshinaga and Masashi Toyoda ............................ 518

Fine-Tuned Transformers Show Clusters of Similar Representations Across Layers

Jason Phang, Haokun Liu and Samuel R. Bowman . . .

BERT Has Uncommon Sense: Similarity Ranking for Word Sense BERTology

Luke Gessler and Nathan Schneider... 



\section{Conference Program}

Thursday, November 11, 2021

\section{Virtual Programme}

\section{2:00-2:15 Opening Remarks}

2:15-3:00 Invited Talk by Jelle Zuidema \& $Q \& A$

3:15-4:00 Oral Session 1

4:30-6:00 Poster Session 1

6:15-7:00 Oral Session 2

7:30-8:00 Invited Talk by Ana Marasović

8:00-8:30 Invited Talk by Sara Hooker

8:30-8:45 Closing 
Thursday, November 11, 2021 (continued)

\title{
Hybrid Programme
}

\author{
9:00-9:15 Opening Remarks \& Best Paper Award \\ 9:15-10:00 Invited Talk by Jelle Zuidema \& $Q \& A$ \\ 10:00-10:30 Oral Session 3 \\ 11:00-12:00 Poster Session 2 \\ 13:00-13:45 Invited Talk by Sara Hooker \& $Q \& A$ \\ 13:45-14:15 Oral Session 4 \\ 14:45-16:15 Poster Session 3 \\ 16:45-17:15 Oral Session 5 \\ 17:15-18:00 Invited Talk by Ana Marasović \& $Q \& A$
}

\section{8:00-18:15 Closing}




\section{Thursday, November 11, 2021 (continued)}

\section{Oral Sessions}

To what extent do human explanations of model behavior align with actual model behavior?

Grusha Prasad, Yixin Nie, Mohit Bansal, Robin Jia, Douwe Kiela and Adina Williams

Test Harder than You Train: Probing with Extrapolation Splits Jenny Kunz and Marco Kuhlmann

Does External Knowledge Help Explainable Natural Language Inference? Automatic Evaluation vs. Human Ratings

Hendrik Schuff, Hsiu-Yu Yang, Heike Adel and Ngoc Thang Vu

The Language Model Understood the Prompt was Ambiguous: Probing Syntactic Uncertainty Through Generation

Laura Aina and Tal Linzen

On the Limits of Minimal Pairs in Contrastive Evaluation

Jannis Vamvas and Rico Sennrich

What Models Know About Their Attackers: Deriving Attacker Information From Latent Representations

Zhouhang Xie, Jonathan Brophy, Adam Noack, Wencong You, Kalyani Asthana, Carter Perkins, Sabrina Reis, Zayd Hammoudeh, Daniel Lowd and Sameer Singh

\section{Poster Sessions}

ALL Dolphins Are Intelligent and SOME Are Friendly: Probing BERT for Nouns' Semantic Properties and their Prototypicality

Marianna Apidianaki and Aina Garí Soler

ProSPer: Probing Human and Neural Network Language Model Understanding of Spatial Perspective

Tessa Masis and Carolyn Anderson

Can Transformers Jump Around Right in Natural Language? Assessing Performance Transfer from SCAN

Rahma Chaabouni, Roberto Dessì and Eugene Kharitonov

Transferring Knowledge from Vision to Language: How to Achieve it and how to Measure it?

Tobias Norlund, Lovisa Hagström and Richard Johansson 


\section{Thursday, November 11, 2021 (continued)}

Discrete representations in neural models of spoken language

Bertrand Higy, Lieke Gelderloos, Afra Alishahi and Grzegorz Chrupała

Word Equations: Inherently Interpretable Sparse Word Embeddings through Sparse Coding

Adly Templeton

A howling success or a working sea? Testing what BERT knows about metaphors Paolo Pedinotti, Eliana Di Palma, Ludovica Cerini and Alessandro Lenci

How Length Prediction Influence the Performance of Non-Autoregressive Translation?

Minghan Wang, GUO Jiaxin, Yuxia Wang, Yimeng Chen, Su Chang, Hengchao Shang, Min Zhang, Shimin Tao and Hao Yang

On the Language-specificity of Multilingual BERT and the Impact of Fine-tuning Marc Tanti, Lonneke van der Plas, Claudia Borg and Albert Gatt

Relating Neural Text Degeneration to Exposure Bias

Ting-Rui Chiang and Yun-Nung Chen

Efficient Explanations from Empirical Explainers

Robert Schwarzenberg, Nils Feldhus and Sebastian Möller

Variation and generality in encoding of syntactic anomaly information in sentence embeddings

Qinxuan Wu and Allyson Ettinger

Enhancing Interpretable Clauses Semantically using Pretrained Word Representation

Rohan Kumar Yadav, Lei Jiao, Ole-Christoffer Granmo and Morten Goodwin

Analyzing BERT's Knowledge of Hypernymy via Prompting

Michael Hanna and David Mareček

An in-depth look at Euclidean disk embeddings for structure preserving parsing

Federico Fancellu, Lan Xiao, Allan Jepson and Afsaneh Fazly

Training Dynamic based data filtering may not work for NLP datasets

Arka Talukdar, Monika Dagar, Prachi Gupta and Varun Menon 


\section{Thursday, November 11, 2021 (continued)}

Multi-Layer Random Perturbation Training for improving Model Generalization Efficiently

Lis Kanashiro Pereira, Yuki Taya and Ichiro Kobayashi

Screening Gender Transfer in Neural Machine Translation

Guillaume Wisniewski, Lichao Zhu, Nicolas Bailler and François Yvon

What BERT Based Language Model Learns in Spoken Transcripts: An Empirical Study

Ayush Kumar, Mukuntha Narayanan Sundararaman and Jithendra Vepa

Assessing the Generalization Capacity of Pre-trained Language Models through Japanese Adversarial Natural Language Inference

Hitomi Yanaka and Koji Mineshima

Investigating Negation in Pre-trained Vision-and-language Models

Radina Dobreva and Frank Keller

Not all parameters are born equal: Attention is mostly what you need

Nikolay Bogoychev

Not All Models Localize Linguistic Knowledge in the Same Place: A Layer-wise Probing on BERToids' Representations

Mohsen Fayyaz, Ehsan Aghazadeh, Ali Modarressi, Hosein Mohebbi and Mohammad Taher Pilehvar

Learning Mathematical Properties of Integers

Maria Ryskina and Kevin Knight

Probing Language Models for Understanding of Temporal Expressions

Shivin Thukral, Kunal Kukreja and Christian Kavouras

How Familiar Does That Sound? Cross-Lingual Representational Similarity Analysis of Acoustic Word Embeddings

Badr Abdullah, Iuliia Zaitova, Tania Avgustinova, Bernd Möbius and Dietrich Klakow

Perturbing Inputs for Fragile Interpretations in Deep Natural Language Processing Sanchit Sinha, Hanjie Chen, Arshdeep Sekhon, Yangfeng Ji and Yanjun Qi

An Investigation of Language Model Interpretability via Sentence Editing Samuel Stevens and $\mathrm{Yu} \mathrm{Su}$ 


\section{Thursday, November 11, 2021 (continued)}

Interacting Knowledge Sources, Inspection and Analysis: Case-studies on Biomedical text processing

Parsa Bagherzadeh and Sabine Bergler

Attacks against Ranking Algorithms with Text Embeddings: A Case Study on Recruitment Algorithms

Anahita Samadi, debapriya banerjee and Shirin Nilizadeh

Controlled tasks for model analysis: Retrieving discrete information from sequences Ionut-Teodor Sorodoc, Gemma Boleda and Marco Baroni

The Acceptability Delta Criterion: Testing Knowledge of Language using the Gradience of Sentence Acceptability

Héctor Vázquez Martínez

How Does BERT Rerank Passages? An Attribution Analysis with Information Bottlenecks

Zhiying Jiang, Raphael Tang, Ji Xin and Jimmy Lin

Do Language Models Know the Way to Rome?

Bastien Liétard, Mostafa Abdou and Anders Søgaard

Exploratory Model Analysis Using Data-Driven Neuron Representations

Daisuke Oba, Naoki Yoshinaga and Masashi Toyoda

Fine-Tuned Transformers Show Clusters of Similar Representations Across Layers Jason Phang, Haokun Liu and Samuel R. Bowman

BERT Has Uncommon Sense: Similarity Ranking for Word Sense BERTology

Luke Gessler and Nathan Schneider 


\section{Thursday, November 11, 2021 (continued)}

\section{Non-archival papers (posters)}

Language Models Use Monotonicity to Assess NPI Licensing - Jaap Jumelet, Milica Denic, Jakub Szymanik, Dieuwke Hupkes and Shane Steinert-Threlkeld

BPE affects Training Data Memorization by Transformer Language Models - Eugene Kharitonov, Marco Baroni and Dieuwke Hupkes

Do contextual language embeddings distinguish between intersective and strictly subsective adjectives? - Michael Goodale and Salvador Mascarenhas

Generalization in neural sequence models: a case study in symbolic mathematics Sean Welleck, Peter West, Jize Cao and Yejin Choi

Human Evaluation Study for Explaining Knowledge Graph Completion - Timo Sztyler and Carolin Lawrence

Transformers Scan both Left and Right - When they Have a Cue - Jan H. Athmer and Denis Paperno

Explaining NLP Models via Minimal Contrastive Editing (MiCE) - Alexis Ross, Ana Marasović and Matthew Peters

Probing structures in the visual region embeddings from multimodal BERT - Victor Milewski, Miryam de Lhoneux and Marie-Francine Moens

Putting Words in BERT's Mouth: Navigating Contextualized Vector Spaces with Pseudowords - Taelin Karidi, Yichu Zhou, Nathan Schneider, Omri Abend and Vivek Srikumar

On Neurons Invariant to Sentence Structural Changes in Neural Machine Translation - Gal Patel, Leshem Choshen and Omri Abend

Explaining Classes through Word Attributions - Samuel Rönnqvist, Amanda Myntti, Aki-Juhani Kyröläinen, Sampo Pyysalo, Veronika Laippala and Filip Ginter

Testing the linguistics of transformer generalizations - Saliha Muradoglu and Mans Hulden 
